\title{
Speckle perception and disturbance limit in laser based projectors
}

\author{
Guy Verschaffelt*a ${ }^{\mathrm{a}}$, Stijn Roelandt ${ }^{\mathrm{b}}$, Youri Meuret ${ }^{\mathrm{b}, \mathrm{c}}$, Wendy Van den Broeck ${ }^{\mathrm{d}}$, Katriina Kilpi ${ }^{\mathrm{d}}$, \\ Bram Lievens $^{\mathrm{d}}$, An Jacobs ${ }^{\mathrm{d}}$, Peter Janssens ${ }^{\mathrm{e}}$, Hugo Thienpont ${ }^{\mathrm{b}}$ \\ a Applied Physics research group (APHY), Vrije Universiteit Brussel, Pleinlaan 2, B-1050 Brussels, \\ Belgium; \\ ${ }^{\mathrm{b}}$ Brussels Photonics Team (B-PHOT), Vrije Universiteit Brussel, Pleinlaan 2, B-1050 Brussels, \\ Belgium; \\ ${ }^{\mathrm{c}}$ Light \& Lighting Laboratory, KU Leuven, Gebroeders De Smetstraat 1, B-9000 Gent, Belgium; \\ diMinds research center for Studies on Media, Information and Telecommunication (SMIT), Vrije \\ Universiteit Brussel, Pleinlaan 9, B-1050 Brussels, Belgium; \\ ${ }^{\mathrm{e}}$ Barco - Projection Division, Noordlaan 5, B-8520 Kuurne, Belgium.
}

\begin{abstract}
We investigate the level of speckle that can be tolerated in a laser cinema projector. For this purpose, we equipped a movie theatre room with a prototype laser projector. A group of 186 participants was gathered to evaluate the speckle perception of several, short movie trailers in a subjective 'Quality of Experience' experiment. This study is important as the introduction of lasers in projection systems has been hampered by the presence of speckle in projected images. We identify a speckle disturbance threshold by statistically analyzing the observers' responses for different values of the amount of speckle, which was monitored using a well-defined speckle measurement method. The analysis shows that the speckle perception of a human observer is not only dependent on the objectively measured amount of speckle, but it is also strongly influenced by the image content. As is also discussed in [Verschaffelt et al., Scientific Reports 5, art. nr. $14105,2015]$ we find that, for moving images, the speckle becomes disturbing if the speckle contrast becomes larger than $6.9 \%$ for the red, $6.0 \%$ for the green, and $4.8 \%$ for the blue primary colors of the projector, whereas for still images the speckle detection threshold is about 3\%. The speckle disturbance limit for movies thus turns out to be substantially larger than that for still images, and hence is easier to attain.
\end{abstract}

Keywords: Speckle, speckle perception, laser projection, image quality.

\section{INTRODUCTION}

Present developments in high-end cinema projection systems are to a large extent focused on further increasing the amount of light projected on the screen. Due to the limitations of arc lamps ${ }^{1}$, the brightness of cinema images projected using a system employing such arc lamps is considered by many people as being too dark ${ }^{2}$. Other disadvantages of arc lamps are the limited lifetime of these lamps ${ }^{3}$ (of typically a few hundred hours) and the gradual decrease of the output power during their lifetime ${ }^{4}$.

Laser display technology is widely considered as the most promising technology for high-lumen future projection applications ${ }^{2,5}$. These sources can combine a high lumen output with a very low étendue, which leads to a high optical efficiency in the projector. The lifetime of a laser is typically one order of magnitude larger than that of arc-lamps, and the output power does not degrade strongly over the laser's lifetime. Laser projectors can therefore potentially outperform current arc-lamp projectors in many different aspects. 
The introduction of lasers in projection systems has been hampered by the presence of speckle in projected images ${ }^{6}$. This speckle pattern is displeasing for a human observer and therefore it needs to be reduced to an acceptable level. Speckle is typically quantified using the speckle contrast $\mathrm{C}$, which is given by the ratio between the standard deviation $\sigma$ of the intensity fluctuations due to speckle and the mean intensity I of the image, i.e.

$$
\mathrm{C}=\frac{\sigma}{I}
$$

Previous studies ${ }^{7}$ resulted in an estimate of the speckle detection threshold for still images at a contrast level of $3 \%$, but hardly any research has been done concerning the level of speckle that can be tolerated when projecting moving images. In this contribution we investigate the speckle perception for moving images in a cinema environment. For this purpose, we equipped a movie theatre room with a prototype laser projector. A group of 186 participants was gathered to evaluate the speckle perception of the moving images in a subjective 'Quality of Experience' (QoE) experiment. The speckle disturbance limit is assessed using several, short movie trailers.

We identify ${ }^{8}$ a speckle disturbance threshold by statistically analyzing the observers' responses for different values of the amount of speckle, which was monitored using a well-defined speckle measurement method. We identify the disturbance threshold by comparing the user responses with those measured previously using a lamp projector (i.e. a projector with no speckle): as long as the quality perception of the laser projector is statistically similar to that of the lamp projector, we consider the speckle contrast of the laser projector as being sufficiently low.

\section{SETUP OF THE PROJECTION SYSTEM}

The laser projection system is placed in the control room at the back of a cinema theatre room. The theatre room is about $30 \mathrm{~m}$ long and has a projection screen with a width of $10 \mathrm{~m}$. The projector has a resolution of $4096 \mathrm{x} 2160$ pixels and its white point is set at the CIE chromaticity diagram coordinates $(0.314,0.351)$. The luminance of the projected images is set at 48 NIT, and the wavelength of the primary colors is $463 \mathrm{~nm}$ for blue, $532 \mathrm{~nm}$ for green and $634 \mathrm{~nm}$ for red. The projection system uses, amongst others, angular decorrelation as a means to reduce speckle. The effect of angular decorrelation is linearly depending on the angular extent of the observer's entrance pupil relative to the screen ${ }^{6}$. This implies that the observed speckle contrast will be smaller if the distance from the observer to the screen increases. Therefore, we will group the participants into 4 different blocks when we perform statistical analysis of the responses. Block 1 corresponds to all the participants seated in rows 3 to 5, Block 2 is the collection of all persons seated in rows 6 to 8 , Block 3 groups all people seated in rows 10 to 12 and Block 4 consists of the participants seated in rows 14 to 16 . Additionally, a polarizer can be placed after the projection lens to further increase the speckle contrast. The polarizer reduces the brightness of the images on the screen, so when no polarizer is used, a neutral density (ND) filter can be used to reduce the brightness of the images (without influencing the speckle contrast) to the same level as when placing a polarizer in the beam's path.

In order to objectively evaluate the amount of speckle experienced by the observers seated at different locations in the theatre room, we have measured the speckle contrast $\mathrm{C}$ for each of the primary colors separately and we performed this measurement at different positions in the room. The speckle contrast $\mathrm{C}$ will depend on the distance to the screen because of the angular speckle decorrelation technique used by the projector. But this measured speckle contrast will also strongly depend on the parameters of the measurement setup ${ }^{9}$, e.g. the measured contrast depends on the pixel size of the CCD camera, on the focal length and f-number of the camera's imaging lens, etc. Therefore, all details of the speckle measurement setup needs to be specified in order to make this measurement reproducible. Moreover, we will employ measurement parameters that are matched to the speckle perception of a human observer.

The measurement setup used to objectively quantify the amount of speckle is based on the findings presented in literature ${ }^{9}$. The speckle contrast values are measured from static images with a uniform intensity distribution. The measurement setup consists of a camera zoom-lens (Nikon Nikkor AF-D 24-85mm) mounted on a 12-bit monochrome CCD camera (Ophir Spiricon SP620U) with a pixel area of $4.40 \times 4.40 \mu \mathrm{m}^{2}$. In order to objectively measure the speckle contrast and define a human speckle disturbance limit, one should make sure the speckle contrast measurement is independent of the projection system and is related to the perception of speckle by a human observer. As a consequence, the camera setting should exhibit a clear aperture of $3.2 \mathrm{~mm}$ and a square root ratio between the pixel area Ap and the speckle area Ac of $\operatorname{Sqrt}(\mathrm{Ap} / \mathrm{Ac})=0.54$. These values are based on the typical characteristics of the human eye, and they 
lead to a pixel size that is small enough such that this pixel size has a negligible effect on the measured speckle contrast ${ }^{9}$. For the pixel area of the used CCD camera, this corresponds to a focal length of the camera lens of $43.4 \mathrm{~mm}$ and an $\mathrm{f}$ number of 13.4. As we can only set the f-number of the used camera lens in discrete steps, we set the f-number to 16 , which is the lens's setting closest to and higher than the desired value. We adjust the focal length to $51 \mathrm{~mm}$ such that the clear aperture of the camera lens remains at $3.2 \mathrm{~mm}$ (being equal to the clear aperture of the human eye at a luminance of $\left.48 \mathrm{NIT}^{10}\right)$. These settings of the f-number and the focal length result in a ratio Sqrt(Ap/Ac) $=0.46$, which is only slightly smaller than the desired value of 0.54 and which leads to a negligible change in the speckle averaging due to the finite pixel size ${ }^{9}$. We make sure that the screen is sharply imaged on the CCD of the camera. Images are acquired in a cinema room with the ambient lighting switched off, resulting in a background ambient light level of about 0.5 NIT. The integration time of the camera is set to $400 \mathrm{~ms}$, which is larger than the 50ms integration time we proposed in literature ${ }^{9}$ This increase in the integration time was needed in order to reduce the noise to an acceptable level, while it should not influence the measured speckle contrast as the laser projector does not use time averaging of a moving speckle pattern as one of the methods to reduce speckle. The electrical camera noise is taken into account by subtracting the (intensity weighted) camera noise from the measured standard deviation of the intensity distribution. Finally, small fluctuations in the intensity across the screen (e.g. due to screen non-uniformities) are typically present at low-spatial frequencies and are removed from the captured images by high-pass spatial filtering.

Using this measurement procedure, we evaluated the speckle contrast at different distances from the screen. The resulting speckle contrast values for the three primary colors (both with polarizer and neutral density filter) are depicted in Fig. 1 as a function of the row, where larger row numbers are located further away from the screen. Note that we have no measurement of the speckle contrast at the first rows of the room, because the measured intensity distribution at these short distances still contained fluctuations due to the finite size of the projector's pixels on the screen. At larger distances, these pixels are no longer resolved on the CCD camera. Also note that the speckle contrast indeed decreases approximately linearly with increasing viewing distance because of angular decorrelation The speckle contrast decreases slightly when no polarizer is placed in the beam path. We will use Fig. 1 in order to link the user responses - grouped in the different blocks - with an objectively measured amount of speckle.

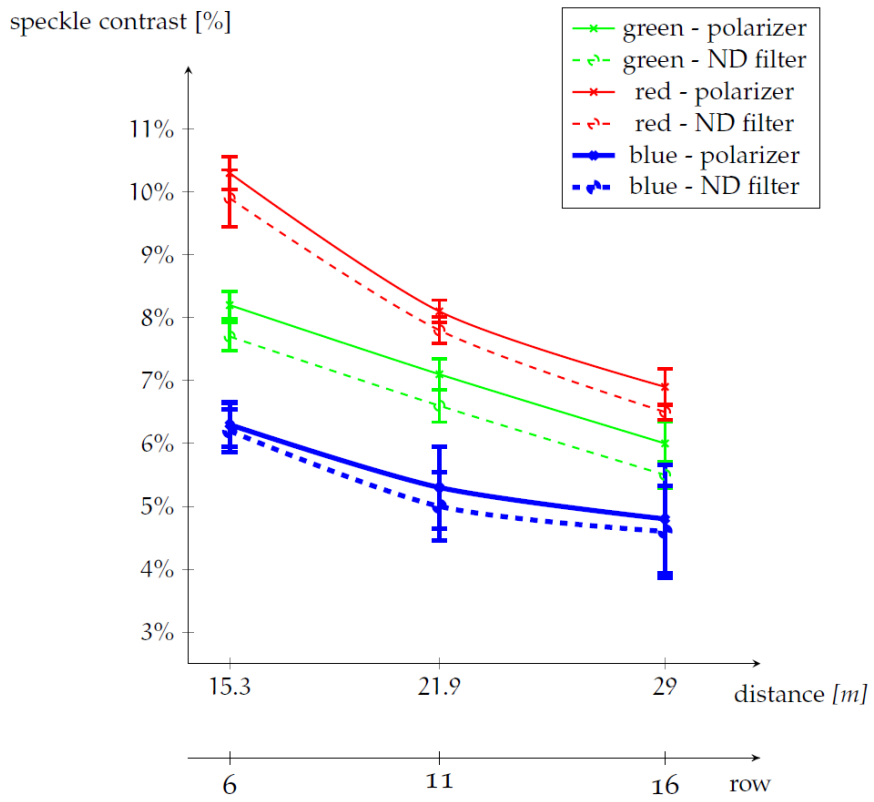

Figure 1. The speckle contrast values of the three primary colors of the laser projector as a function of the distance from the screen in the cinema theatre room. At the bottom we also indicate the position of different rows. Each speckle contrast is the average of about 20 measurements. The symbols are the actual averaged measurement points, the lines are included to guide the eye. The solid lines correspond to the speckle contrast values when a polarizer is placed in front of the projection system and the dashed lines represent the speckle contrast values when a neutral density filter is placed in front of the projection system. Vertical bars correspond to the standard deviation of the measured speckle contrast values. 


\section{PERCEPTION OF SPECKLE}

A total of 186 persons participated in the test, which were almost evenly distributed over the different blocks in the room. In an initial phase of the test, speckle was explained to the participants using some images in which we artificially embedded a speckle-like granularity in the image's intensity distribution. To make respondents familiar with the used voting system for the test, some simple questions were asked (e.g. how did you get here, gender, color blindness). Afterwards, six different trailers are shown, three trailers of animated movies (Monsters University, Hotel Transsylvania, Ice Age 4) and three trailers of regular movies with different content (Man of Steel, Bourne Legacy, Twilight Saga: Breaking Dawn). The trailers are shown with a polarizer, a neutral density filter or neither. After every trailer, the participants are asked to evaluate the speckle quality with a score ranging from 1 (imperceptible) to 5 (very disturbing). The exact grading of the scores is shown in Table 1. After the trailers, the participants are shown a full-length movie called TED while they are unaware of the fact that the movie is shown using a laser projector and that this movie is still part of the experiment. The movie is presented as a reward for participating in the test and provides respondents with a realistic cinema experience. After the full-length movie, the participants are again asked to evaluate the amount of speckle using the same scale as before.

Table 1: Table showing the grading of the scores for speckle perception in the questionnaire.

\begin{tabular}{|l|l|}
\hline Mark for the images how you see speckle \\
\hline Imperceptible & 1 \\
\hline Perceptible, but not annoying & 2 \\
\hline Slightly annoying & 3 \\
\hline Annoying & 4 \\
\hline Very annoying & 5 \\
\hline
\end{tabular}

Starting from the users' responses we determine, for every movie trailer, the percentage of people that noticed speckle and the Mean Opinion Score (MOS) on the scale expressed in Table 1. From this analysis, it becomes clear that the movie content exerts an influence on the perception of speckle. The percentage of people that noticed speckle in the Monsters University trailer is only 3\% and is about 4 times lower than in the Breaking Dawn trailer, for which $13 \%$ of the participants notice speckle. Also the speckle MOS is clearly higher for the Breaking Dawn trailer as compared to the Monsters University trailer. Nevertheless, both trailers have been shown with exactly the same projector settings, resulting in identical amounts of speckle in the projected images. The speckle MOS is typically higher for those trailers that contain large grey areas (e.g. from a wall or a cloudy sky) with a rather uniform intensity distribution and that change slowly in time.

\section{SPECKLE DISTURBANCE LIMIT}

We further investigate the speckle disturbance limit by evaluating the responses concerning the Man of Steel trailer as the percentage of people observing speckle and the speckle MOS are highest for this trailer as compared to the other trailers. Therefore, selecting this trailer for further analysis corresponds to taking the worst case with respect to speckle perception.

There is no clear threshold for the observation of speckle: some people will observe speckle even when the speckle contrast is very low, while other people do not see it at high speckle contrast values. Therefore, we have chosen to set this threshold based on the perception of speckle in still images using a lamp projector that we know from a previous study $^{7}$ : when we asked a test audience to evaluate the speckle perception of still images in a lamp projector, this resulted in a speckle MOS of 1.5 on the scale defined by Table 1. The objectively measured speckle contrast of this lamp projector is close to zero, but the audience in the test did not know when they were evaluating a laser projector and when they were looking at images projected with a lamp projector. Therefore, if the speckle MOS of the laser projector is below 1.5, this indicates that people perceive the same amount of speckle as for a lamp projector, and there is no need to try to further lower the speckle MOS below 1.5. For that reason we set the speckle disturbance limit as the objectively measured speckle contrast at which a speckle MOS of 1.5 is reached. 
The distribution of the observers score's for the speckle perception is not Gaussian. This is illustrated in Fig. 2, where we plot all user responses when the laser projector is used to show the Bourne Legacy trailer. The solid curve is a normal distribution fit based on the speckle MOS and the standard deviation of the observer scores. This curve does not match well with the histogram of the observer scores. Similarly shaped distributions of the observer scores are also found for the other trailers. Therefore, we have to make use of a non-parametric statistical test in order to analyze the data.

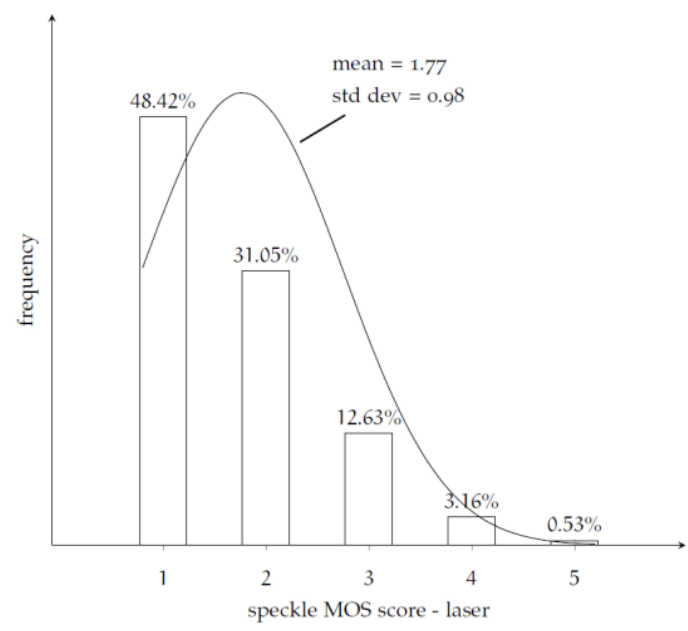

Figure 2. Histogram of the speckle score of all user responses when the laser projector is used to show the Bourne Legacy trailer.

We have used a non-parametric Kruskal-Wallis test ${ }^{11}$ to identify statistically significant differences between the responses of the people sitting in different blocks when considering the Man of Steel trailer. The results of this analysis are summarized in Table 2, in which we show the number of responses N, the speckle MOS for each block and the mean difference in speckle scores between the blocks. From this table, it can be deduced that the speckle MOS decreases as people are seated further away from the screen. This reduction of the speckle MOS is due to the decrease in the amount of speckle observed in Fig. 1 when the distance to the screen is increased. It is important to note that the speckle MOS is slightly below 1.5 for the last block, i.e. for Block 4 . The position corresponding to the speckle disturbance limit can thus be located between Block 3 and Block 4 .

Table 2: Analysis of speckle Mean Opinion Score (MOS) and mean difference in speckle score of the Man of Steel trailer, and how these quantities depend on the block number. $\mathrm{N}$ is the total number of observations within each data set.

\begin{tabular}{|c|c|c|c|c|c|c|}
\hline & & & & an differ & speckle & \\
\hline & $\mathrm{N}$ & $\begin{array}{l}\text { Speckle } \\
\text { MOS }\end{array}$ & Block 1 & Block 2 & Block 3 & Block 4 \\
\hline Block 1 & 46 & 1.98 & - & -0.24 & -0.23 & $-0.57^{* * *}$ \\
\hline Block 2 & 42 & 1.74 & & - & 0.01 & $-0.33^{*}$ \\
\hline Block 3 & 43 & 1.74 & & & - & $-0.33^{* *}$ \\
\hline Block 4 & 51 & 1.41 & & & & - \\
\hline
\end{tabular}

Looking at the mean difference in speckle score, it is clear that Block 4 is statistically significantly different from all the other blocks. This implies that there is a clear difference in speckle perception between the third and the fourth block. The speckle MOS decreases from 1.74 to 1.41. As we aim to achieve a speckle MOS smaller than 1.5 (because this is equivalent to the speckle perception in a lamp projector), the speckle disturbance limit will be close to the speckle contrast reported in Fig. 1 for row 16 with polarizer, i.e. the speckle disturbance limit for moving images is reached in our setup for $\mathrm{C}=(6.9 \pm 0.3) \%$ for red, $\mathrm{C}=(6.0 \pm 0.3) \%$ for green, and $\mathrm{C}=(4.8 \pm 0.9) \%$ for blue. 


\section{FULL LENGTH MOVIE TEST}

After the speckle perception tests with the movie trailers, the participants were rewarded by showing them the movie TED. What they did not know was that the movie itself was projected using a laser projector. Furthermore, the speckle settings were different before and after the break. Before the break, a polarizer was placed in front of the projection system, while after the break, the neutral density filter was used. As the participants were not aware of the additional experiment, their primary focus was on the content, rather than on identifying speckle.

After the movie, the participants were asked whether they noticed speckle in the movie. A majority of the people mentioned that speckle was imperceptible $(69 \%)$, followed by $29.0 \%$ of the participants mentioning that speckle was perceptible, but not annoying. The remaining $2 \%$ rates the speckle quality slightly annoying to very annoying. That means that $98 \%$ of the participants was watching a movie - projected by a prototype laser projector- and was not disturbed by the fact that there was (a relatively high amount of) speckle in the images (with a speckle contrast up to 8 $10 \%$ depending on the color and the position in the room).

Also for this full length movie, we analyzed the user responses and investigated whether or not the speckle perception is dependent on different parameters. In table 3 we show the effect of the distance to the screen on the speckle perception of the full length movie, where we again have grouped the responses in different blocks as described before. In this table we specify the speckle MOS, the mean difference in the speckle MOS score for the different blocks, and we indicate which differences are statistically significant. The speckle MOS of the full length movie is clearly lower than for the Man Of Steel-trailer, indicating that the latter probably corresponds to the worst case scenario when considering speckle perception. For the full length movie, the speckle MOS always remains smaller than 1.5, even for the first rows, indicating that the amount of speckle is sufficiently low not to be disturbing in the full length movie experience. The speckle perception in Table 3 slightly depends on the block number, and again the speckle MOS decreases with increasing distance to the screen which is consistent with the measured amount of speckle shown in Fig. 1.

Table 3: Analysis of speckle Mean Opinion Score (MOS) and mean difference in speckle score of the full length TED movie, and how these quantities depend on the block number. $\mathrm{N}$ is the total number of observations within each data set.

\begin{tabular}{|c|c|c|c|c|c|c|}
\hline & & & & an differ & speckle & \\
\hline & $\mathrm{N}$ & $\begin{array}{l}\text { Speckle } \\
\text { MOS }\end{array}$ & Block 1 & Block 2 & Block 3 & Block 4 \\
\hline Block 1 & 46 & 1.47 & - & -0.18 & -0.02 & $-0.31^{* * 1}$ \\
\hline Block 2 & 42 & 1.29 & & - & 0.16 & -0.13 \\
\hline Block 3 & 43 & 1.45 & & & - & $-0.29^{* * *}$ \\
\hline Block 4 & 51 & 1.16 & & & & - \\
\hline
\end{tabular}

Furthermore, when the participants were asked whether they noticed a difference in the amount of speckle before and after the break, $88.6 \%$ did not notice a difference. For the remaining part, $5.8 \%$ preferred the image quality before the break (with the polarizer and thus higher speckle) and the remaining $5.3 \%$ preferred the image quality after the break. Therefore, there was no significant difference in the quality perception with and without the polarizer and the corresponding small difference in speckle contrast (see Fig. 1) is insufficient to be noticed.

Together with the users' evaluation of the perceived amount of speckle during the full length movie, we also asked a number of supplementary questions about the participants and their movie experience. We inquired the participants about their age, gender, if they have color blindness, and if they wear glasses. The result of this statistical analysis is presented in Table 4. None of these parameters seem to have a significant influence on the perception of speckle. 
Table 4: Analysis of speckle Mean Opinion Score (MOS) and mean difference in speckle score of the full length TED movie, and how these quantities depend on the participants' gender, color blindness and their use of glasses. $\mathrm{N}$ is the total number of observations within each data set.

\begin{tabular}{|l|c|c|c|c|}
\hline \multicolumn{2}{|l|}{ Gender? } & \multicolumn{2}{c|}{ Mean difference speckle score } \\
\hline & $\mathrm{N}$ & Speckle MOS & Male & Female \\
\hline Male & 95 & 1.34 & - & -0.01 \\
\hline Female & 88 & 1.33 & & - \\
\hline
\end{tabular}

\begin{tabular}{|l|c|c|c|c|}
\hline \multicolumn{2}{|c|}{ Color Blindness? } & \multicolumn{2}{c|}{ Mean difference speckle score } \\
\hline & $\mathrm{N}$ & Speckle MOS & Yes & No \\
\hline Yes & 6 & 1.33 & - & 0.02 \\
\hline No & 170 & 1.35 & & - \\
\hline
\end{tabular}

\begin{tabular}{|l|c|c|c|c|c|}
\hline Wearing glasses? & \multicolumn{3}{c|}{ Mean difference speckle score } \\
\hline & $\mathrm{N}$ & Speckle MOS & Yes & No & Sometimes \\
\hline Yes & 44 & 1.43 & - & -0.08 & -0.24 \\
\hline No & 89 & 1.35 & & - & -0.16 \\
\hline Sometimes & 42 & 1.19 & & & - \\
\hline
\end{tabular}

We also asked some contextual questions about the movie going experience of the participants, for example we asked whether or not they had something to eat or drink and we asked if they have been talking to other participants during the full length movie. The results of this statistical analysis are presented in Table 5. We did not find significant influences of the investigated contextual factors on the perception of speckle.

Table 5: Analysis of speckle Mean Opinion Score (MOS) and mean difference in speckle score of the full length TED movie, and how these quantities depend on the contextual factors of the movie going experience. $\mathrm{N}$ is the total number of observations within each data set.

\begin{tabular}{|l|c|c|c|c|}
\hline \multicolumn{2}{|c|}{ Did you eat/drink during movie? } & \multicolumn{2}{c|}{ Mean difference speckle score } \\
\hline & $\mathrm{N}$ & Speckle MOS & Yes & No \\
\hline Yes & 142 & 1.32 & - & 0.01 \\
\hline No & 9 & 1.33 & & - \\
\hline
\end{tabular}

\begin{tabular}{|l|c|c|c|c|}
\hline \multicolumn{2}{|c|}{ Did you speak during movie? } & \multicolumn{2}{c|}{ Mean difference speckle score } \\
\hline & $\mathrm{N}$ & Speckle MOS & Yes & No \\
\hline Yes & 6 & 1.36 & - & -0.07 \\
\hline No & 170 & 1.29 & & - \\
\hline
\end{tabular}

\section{CONCLUSIONS}

The speckle disturbance limit in the case of moving images turns out to be much higher than for still images. In a previous study ${ }^{7}$ we used a similar procedure as the one described here to estimate the speckle perception of still images, leading to a speckle disturbance limit being equal to the speckle detection limit of $3.6 \%$ for the red $(639 \mathrm{~nm}), \mathrm{C}=3.2 \%$ for the green $(532 \mathrm{~nm})$ and $\mathrm{C}=4.4 \%$ for the blue $(465 \mathrm{~nm})$ primary colors. In the study presented here using moving images $^{8}$, we could not independently change the speckle contrast of each of the primary colors. Therefore, it is possible that the measured speckle disturbance limit is mainly dominated by the speckle disturbance caused by one of the primary colors, and that the speckle disturbance limit of the other colors is actually (slightly) larger. As the speckle contrast is always largest for the red primary color in our projector setup, it is thus probable that the disturbance limit is set by the speckle at the red wavelength channel. We have also observed that the speckle perception is influenced by the content of 
the movie, but not by other factors such as age or gender. We find that, when considering the trailer that corresponds to the strongest speckle perception by the participants, the speckle becomes disturbing in moving images if the speckle contrast becomes larger than $(6.9 \pm 0.3)$ for the red, $(6.0 \pm 0.3) \%$ for the green, and $(4.8 \pm 0.9) \%$ for the blue primary colors of the projector.

\section{REFERENCES}

[1] Derra, G., Moench, H., Fischer, E., Giese, H., Hechtfischer, U., Heusler, G., Koerber, A., Niemann, U., Noertemann,F.-C., Pekarski, P., Pollmann-Retsch, J., Ritz A. and Weichmann, U. "UHP lamp systems for projection applications," J. Phys. D: Appl. Phys. 38, 2995-3010 (2005).

[2] Beck, B., "Lasers light up the silver screen," IEEE Spectrum 51, 32-39 (2014).

[3] Brennesholtz, M.S. and Stupp, E.H., [Projection Displays], Wiley \& Sons (2008).

[4] Brennesholtz, M.S., "New-Technology Light Sources for Projection Displays," SID Int. Symp. Digest of Technical Papers 39, 858-861 (2008).

[5] Chellappan, K., Erden, E. and Urey, H. , “Laser-based displays: a review,” Appl. Opt. 49, 79-98 (2010).

[6] Goodman, J.W., [Speckle Phenomena in Optics: Theory and Applications], Roberts and Company (2007).

[7] Roelandt, S., Meuret, Y., Jacobs, A., Willaert, K., Janssens, P., Thienpont, H. and Verschaffelt, G., "Human speckle perception threshold for still images from a laser projection system", Opt. Express 22, 23965-23979 (2014).

[8] Verschaffelt, G., Roelandt, S., Meuret, Y., Van den Broeck, W., Kilpi, K., Lievens, B., Jacobs, A., Janssens, P. and Thienpont, H., "Speckle disturbance limit in laser-based cinema projection systems," Scientific Reports 5 , art. nr. 14105 (2015).

[9] Roelandt, S., Meuret, Y., Craggs, G., Verschaffelt, G., Janssens, P. and Thienpont, H., "Standardized speckle measurement method matched to human speckle perception in laser projection systems", Opt. Express 20, 8770-8783 (2012).

[10] Society of Motion Picture and Television Engineers, "SMPTE standard RP431-2:2011: D-Cinema Quality Reference Projector and Environment," <http://standards.smpte.org/content/978-1-61482-243-1/rp-431-22011/SEC1.body.pdf+html> (26 February 2015).

[11] Kruskal, W.H. and Wallis, W.A., "Use of ranks in one-criterion variance analysis," Journal of the American statistical Association 47, 583-621 (1952). 